\title{
Maritime Infrared Background Clutter
}

\author{
Piet Schwering \\ TNO Physics and Electronics Laboratory \\ P.O. Box 96864, NL-2509 JC The Hague, The Netherlands \\ E-mail: Schwering@fel.tno.nl
}

\begin{abstract}
The detection of small targets in maritime infrared surveillance is hampered by the presence of clutter. Sea surface structure, reflection and emission changes related to inciclent angle variations and surface effects are standard features governing the clutter behavior. Also special effects as sun glint and horizon effects play an important role for clutter. In order to optimize the detection process, quantitative clutter estimates are of use for filter settings. We have recorded a large amount of infrared backgrounds in the last few years, during common NATO trials. A large amount of different meteorological conditions took place during the various experiments. A first set of these data have been analyzed to obtain statistical data that represent the infrared scene. We have derived vertical temperature profiles, vertical fluctuation profiles, horizontal correlation coefficients and temporal correlation functions. In this paper we present the first analysis of these data. We are in the process of obtaining a condensed database of information to regenerate clutter images from bulk meteo parameters, and clutter parameters. The clutter and meteo parameters have been used to simulate various infrared scenes. Examples of this simulation process are shown in the presentation. The simulated images are statistically similar to the original images that were used to derive the parameters. A description of the image re-generation is presented. Future expansions of the model are discussed.
\end{abstract}

\section{INTRODUCTION}

In the last decades infrared surveillance systems in naval scenarios have become a major area of interest for research. For the detection of missiles and other objects, as surface objects, these systems can be of great use during day and night operations. Unfortunately, infrared surveillance systems are hampered by clutter caused by various physical objects and structures.

There are two main effects that are of interest for the system, detection and clutter rejection. The system performance can be characterized by the Receiver Operating Characteristics giving the relation between the probability of detection and the probability of false alarms. In order to maximize the detection probability the false alarm probability due to clutter is increased, and visa versa. Obviously, infrared surveillance systems should maximize the detection probability while minimizing the false alarm probability. In practice, a lower limit of the probability of detection is combined with an acceptable upper limit of the false alarm probability.

In order to derive system performances for infrared surveillance systems, live trials are needed. For various reasons, cost, availability and statistics of scenario, etc. live trials can not always be performed. For instance, instead of probabilities, that can only be obtained by numerous identical trials, only low- 
number statistical samples are taken. For extrapolation of the performance of systems in other scenarios, no experimental data is available normally. Therefore system modeling is of the greatest importance. A system model predicts the performance of the system, while input data is generated for it representing the scenario. During the mentioned live trials, some experimental data is coupled to the model, and the model is validated during those trials. The extrapolation of the system into other scenarios is then done by extrapolating the model input scene. This model input scene consists of three component, targets, background and atmosphere.

In this paper we describe a method for determining the background scene in a naval scenario. In the next section we will summarize the clutter importance due to the various physical components. In section 3 we continue with a description of trials and a the method for derivation of the model parameters from the data gathered. In section 4 we describe the method used by us to simulate the background structures in the scene. Section 5 gives some first results of the background scene generation together with a comparison between generated and original data. Section 6 gives the preliminary conclusions of this paper and suggestions for further work.

\section{ANALYSIS OF MARITIME BACKGROUND PROPERTIES}

During the last ten years a number of papers have appeared on maritime infrared backgrounds and on the analysis and modeling of the naval background. Especially in the NATO community the importance of accurate maritime background information has been expressed. The fact the various NATO trials have been undertaken to investigate backgrounds (SIVEX 1989, MAPTIP 1993), and the fact that more trials are proposed (LAPTEX 1996) shows that infrared backgrounds still are an area of interest in the community.

In this section we describe the effects that play a role in the naval infrared background. As this background we define the photons as they arrive on the lens of the sensor system. For the background scene we want to have both the local average infrared radiation as well as the true spatial and temporal structure present. For this reason we list the main features that are of interest in the maritime scenario. A number of different background features can be distinguished. We list these features with their respective literature.

- Empty sky background: These are the ideal situations for an infrared system working in the maritime environment. The reason is that no structure is present, and therefor all structure seen in the imager is caused by system noise. The system is then noise limited, and the theoretical system detection limit is valid. From the statistical point of view it is of interest to know the occurrence of empty sky. In the background model, the average background values, determines the infrared contrast. The spectral behavior of the background can be different in various situations, especially with solar scattering in the 3-5 micron band. MODTRAN (AFGL 19\$9, [1]) can be used to predict sky background radiance.

- Cloudy sky background: Clouds can be considered water compartments in the sky at the temperature approximating that of the surrounding air. As these clouds are optically thick in the infrared wavelength bands, clouds immediately show up as warm objects with respect to the cold sky background. At the cloudy edges sharp contrast jumps occur from the cloud to the empty background. High-pass filters designed for the detection of small targets will enhance these clutter edges. The first difference compared to empty sky is that the background level will be somewhat higher, and hence lowering the target contrast (for targets somewhat warmer than the sky). The second difference is that due to sharp edges more clutter is present, which could increase the detection threshold, or could give rise to erroneous tracks by association with clutter plots. For studies on the cloudy sky backgrounds we refer to Sichwering [2] and Schwering and Kruseman [3], [4]. Levesque's Cloud model (DREV, [5]) creates a cloud and sky background without horizon, in a wicle infrared band.

- Sea background: Sea backgrounds are of importance for targets that have an altitude lower than the 
observing system. Surface and low flying targets are seen against a sea background. The sea background contains two components in the infrared. First of all the emission by the sea surface, caused by it's temperature, and second reflections of ambient radiation (air temperature). The balance depends on the emission and reflection coefficients. Hence under grazing angles the reflecting part is significant. Beside this standard scenario, irregular patterns occur. White caps and irregularities at wave tops can change the coefficients, showing complete internal reflection. The reflecting part of the emission depends on the direction where the emission is coming from. Therefore the wave structure of the sea is of importance. Knowledge of the wave slope statistics dates from Cox and Munk $(1954,1956)$. Data on the interaction probability density for waves is available from Zeisse $(1994,1995)$. It may be clear that the sea state can have a large influence on the intensities received from the sea. It is very complicated to describe the entire effect to sea state only. For studies on sea backgrounds we refer to Schwering [6]. The statistical properties of the sea background are the spatial and temporal correlation coefficients. These factors contain a much wider range than for the cloudy sky background. Models as the Levesque's Sea model (DREV, [7]), SeaRad (NSSOSC/NRaD, [8]), Seascape (Johns Hopkins APL, [9]), model the sea background via different approaches.

- Sun glint effects: Normally solar reflections on the sea surface (sun glint of the surface) are seen as a solar reflection path in elevation with a broad azimuthal extend of several degrees on the sea surface. Solar reflections on the surface near the horizon cause a small constant elevation band over a wide range in azimuth. Solar reflections on cloud edges lead to sunlit clouds and forward solar scattering can be observed in the air. Due to the fact that solar reflections will dominate the image, the background level as well as the clutter level will go up. This has a negative influence in the detection process for detecting small targets. These cases can be considered as special cases of the influence of the sun on sea, cloud and empty sky backgrounds. We refer to Schwering [6] for a spectral study on sun glint. Sun glint is considered in the models mentioned under sea background.

- Horizon effects: As we are searching targets at long ranges they appear near the horizon. The horizon is a discontinuity in temperature slope, the presence of the horizon can degrade the performance of an surveillance system compared to the performance in the empty sky background. Following Saunders [10] multiple reflections and shadowing are important additions to the calculations performed with the Cox-Munk wave slope statistics in order to calculate the sea background radiance in the infrared. Each of these effects become much more important if the source or the receiver is close to the horizon. From the Cox-Munk statistics infinities are predicted near the horizon. However for calm seas no realistic radiance values are obtained. By neglecting multiple reflections and shadowing effects, Zeisse [11], [12] studied the radiance at the ocean horizon by applying an integral equation for all geometries. Finite and proper values are obtained with sun glints and with calm sea as well. Wave structures near the horizon can occur. Additionally sun glint effects near the horizon can be very different from sun glint effects in the open sea.

Many efforts have gone in the spatial and temporal clutter classification by applying correlation filters. The amount of data for spectral background information is limited. Beside spectral data of sun glints and forward scattered solar radiation not much spectral differences are expected in the medium and long wave infrared background. Targets that are above the sea level will show up against the horizon, then against the sky (and cloud) background, and when it closes in and if it is below the height of the sensor, again against the horizon and finally against the sea background. Therefore all these backgrounds are of interest. Sun glints have different effects on the background, such as forward scattering in the sky and reflection on the sea surface. Via a model as IRTool (ONR/Arete, [1:3]) a complete scene can be simulated.

The results that can be derived so far is that none of the existing models is accurate or reliable enough around the horizon. It has been decided that at present, although a preferred situation in the long run is a fully theoretical approach, a completely theoretical approach will not give us the requested accuracy in the model in the short-term time frame, especially near the horizon. Images obtained by us also show 
temperature jumps near the horizon that are not smooth at all (see section 5). We decided to perform an infrared model of the background in broad infrared bands based on empirical data. The model will then be a semi-empirical model, based on scene structures clerived from image sequences taken in different scenarios (different meteorological scenario parameters). The main emphasis is on accuracy of radiance values near the horizon under normal conditions. Special conditions as sun glint will be incorporated at a later stage. The development of this empirical model should also provide us with more knowledge, and therefore assist with the longer term modeling based on physics.

\section{DERIVATION OF BACKGROUND PARAMETERS}

The simulation model MIBS (Maritime Infrared Background Simulator) has been evolved using data gathered by TNO-FEL in the last few years. In this phase of the project North sea (MAPTIP [14]) and Atlantic Ocean data [15], obtained from measurements on-board a ship, have been used to derive the input parameters which are used in the background model to generate the images. Due to the limited amount of time the analyzed images are for the 10 micron band only. Therefore this version of the model works in the long infrared wave band only. Emphasis is put also on the cool and humid atmospheres. The following parameters are used in the model:

- The average value (AVG) of the temperature as a function of elevation, which is contained in a file 'profile\#.dat' (512 points).

- An RMS value of air temperature and one for sea temperature.

- A horizontal and vertical correlation length in air, at the horizon and at the detector position. In the model the correlation length at sea is a linear interpolation of the values at the horizon and the sensor position.

- A threshold value for generating an asymmetrical density distribution (see section 4).

- A multiplying value for modifying the Gaussian probability density function (see section 4).

When we assume that the simulations must be performed with a certain resolution, the image data needed to derive the parameters should be of a better spatial and temporal resolution. A similar reasoning holds for the observation height with respect to the simulation heights, and for all sensor parameters and artifacts. A full de-convolution would be needed. Because of vignetting effects of the USFA UA-92 camera used at both trials only the central part of the images is used to derive the parameters. Therefore the useful field of view of the images is $3{ }_{v}^{\circ} x 1{ }_{h}^{\circ}$. The average value and RMS value of temperature as a function of elevation (called the horizontal profile) are obtained using standard statistical operations. These operations are implemented in a computer program which determines the vertical profile of a given scene. No unambiguous method is found yet to determine correlation length in vertical and horizontal direction as a function of elevation of a scene, and to determine the threshold and the multiplication factor needed to generate an asymmetrical probability density function.

Table 1: Meteorological data of the scenes analyzed at present in the model.

\begin{tabular}{lllllll}
\hline Image & $T_{\text {air }}\left({ }^{\circ} \mathrm{C}\right)$ & $T_{\text {sea }}\left({ }^{\circ} \mathrm{C}\right)$ & $R H(\%)$ & $p$ (mbar) & $v_{\text {wind }}(\mathrm{kts})$ & Dir wind $($ EoN) \\
\hline 08 B5001 & 26.3 & 28.2 & 85.0 & 1020.5 & 11.0 & 050 \\
264001 & 11.0 & 11.5 & 78.3 & 1031.5 & 6.3 & 039 \\
271 L0030 & 10.5 & 11.5 & 87.0 & 1030.0 & 6.4 & 047 \\
$2610-201$ & 10.0 & 11.5 & 83.0 & 1031.0 & 5.8 & 048 \\
12B5001 & 22.8 & 22.2 & 87.0 & 1018.6 & 20 & 215 \\
\hline
\end{tabular}


At this stage five different scenes are used to derive the parameters for image generation. The meteorological data of these scenes is listed in table 1. The two humid scenario measurements were taken during the Atlantic Ocean trial in the Carribean.

\section{BACKGROUND IMAGE SIMULATION}

After analyzing the infrared images, we must choose a method to re-generate the infrared imagery. The objective is to obtain an image that is as close as possible to the original infrared image in radiance values and in structure. At first several methods were investigated to generate background images. These methods are :

- sampling from the obtained statistics (mean value and RMS).

- spatially correlated simulation.

- Markov random fields texture model.

The above methods were tested and produce the right image (vertical profile) with respect to the input data. In the first method the temperature $T$ of points in the image is obtained using a normal random number generation scheme [16]. In this case adjacent points are uncorrelated and typical clutter structures are not generated in the image. The second method uses normal random number generation with spatial correlation [17],[18]. The correlation function is given by $\langle T(R+r) * T(r)\rangle=\sigma^{2} \exp (-\alpha r)$, where $r$ is the relative position of two points, $\alpha^{-1}$ the correlation length, $\sigma^{2}$ the temperature variance and the $\langle\ldots\rangle$ brackets signify a scene average over $R$ (position). In this way synthetic structures can be produced in the horizontal and vertical direction. The Markov random fields method is a probabilistic method for texture modeling [19]. Although promising the implementation of this method is elaborate and the generation of images will take a lot of computer processing time, especially when a large number of gray values are present in the scene. For that reason the attention in this work is focused on generating background images using spatial correlated simulation.

In this model using spatial correlation, the statistical properties of the temperature $T$ over a given scene are described by a normal distribution with certain average value and standard deviation. The average value of $T$, as a function of elevation, is obtained from real imagery, see section 3 . For the standard deviation the average RMS values in air and in sea are used. The multivariate probability density function $P\left(T_{1}, T_{2} \ldots T_{N} ; r_{1}, r_{2} \ldots r_{N}\right)$ (the probability of finding $T_{1}$ at $r_{1}, T_{2}$ at $r_{2}$, etc. and $T_{N}$ at $r_{N}$ ) can be written explicitly as $P(\beta)=\operatorname{det}(V)^{-1 / 2}(2 \pi)^{-N / 2} \exp \left(-\frac{1}{2} \beta^{T} V^{-1} \beta\right)$ where $\beta=\left(T_{1}, T_{2} \ldots T_{N}\right), \beta^{T}$ is the transposed vector, and $V$ is the covariance matrix $V=\left\{\left\langle T_{i} * T_{j}\right\rangle\right\}$ containing the correlation function [17]. For constructing a scene, the conditional probability $P\left(T_{m} \mid T_{1} \ldots T_{K}\right.$ ) (the probability of $T_{m}$ given $T_{1} \ldots T_{K}$ ) is used, which can be obtained from $P(\beta)$. It follows that, given $T_{1} \ldots T_{K}$, then $T_{m}$ is also normally distributed with average and standard deviation depending on $T_{1} \ldots T_{K}$ and $V$. Therefore, $T_{m}$ can be obtained using any normal distributed random number scheme [16].

The spatial correlation is introduced to generate structures. In real images the correlation length depends on elevation. In the model the horizontal and vertical correlation lengths are interpolated values between horizon and sensor position. At this stage simple linear interpolation is used. A consequence of using spatial correlation is that the RMS-value of the generated images decreases with increasing correlation length. For that reason the input value for the RMS in the model has to be adapted (slightly higher) to end up with a realistic RMS-value for the generated image (similar to the one in the original image).

Histograms of real images containing clutter in maritime environments reveal a long-tailed nature of the intensities and suggest that the probability density function is more like a Gamma-distribution. An artificial adaptation in the Gaussian distribution of the model is therefore made to generate an asymmetrical density 
function. This is done by modifying the generated value from the normal clistribution when this value exceeds a certain threshold (this implies that the normalization of the used density function has to be modified, at this stage of the model however this is not done).

\section{IMAGE GENERATION EXAMPLES AND DISCUSSION}

The process to generate background images is implemented in a computer program 'MIBS'. This program, which is in an experimental version now, produces a file 'TEMP.DAT' containing the temperatures of points of a particular generated scene. At present a choice between five different scenes can be made. The total field of view of the scene is 3 degrees in azimuth and 3 degrees in elevation (lattice $512 \times 512$ points). The temperature values are stored as 16 bit values ( 2 bytes), the total size of the file is 524.288 Kbytes. The temperature in degrees Centigrade for each point of the generated scene can be obtained from the file using the following relation : $T=(256 *$ HighByte + Low Bytc $) / 1000$. An easier output format will be chosen in the near future. Five different scenarios are simulated with the model using the input parameters listed in Table 2.

Table 2: Input parameters derived from the scenes at present.

\begin{tabular}{|c|c|c|c|c|c|}
\hline Image & 08B5001 & 264001 & $271 \mathrm{~L} 0030$ & $2610-201$ & $12 \mathrm{~B} 5001$ \\
\hline Average value of temperature & profile1.dat & profile 2. dat & profile:3.dat & profile4.dat & profile5.dat \\
\hline RMS air & 5.0 & 4.0 & 3.0 & 7.0 & 7.0 \\
\hline RMS sea & 10.0 & 7.0 & 4.0 & 10.0 & 14.0 \\
\hline \multicolumn{6}{|l|}{ Correlation length in air: } \\
\hline - horizontal & 2 & 2 & 0.5 & 0.5 & 2 \\
\hline - vertical & 0.5 & 0.5 & 0.5 & 0.5 & 0.5 \\
\hline \multicolumn{6}{|l|}{ Correlation length at horizon: } \\
\hline - horizontal & 4 & 3 & 0.5 & 2 & 4 \\
\hline - vertical & 1 & 1 & 0.5 & 1 & 1 \\
\hline \multicolumn{6}{|c|}{ Correlation length at sensorposition: } \\
\hline - horizontal & 12 & 6 & 10 & 10 & 12 \\
\hline - vertical & 5 & 3 & 4 & 3 & 5 \\
\hline \multicolumn{6}{|l|}{ Clutter parameters: } \\
\hline Threshold & $1.3 \sigma$ & $1.3 \sigma$ & $1.3 \sigma$ & $1.3 \sigma$ & $1.3 \sigma$ \\
\hline Multiplying factor: & 2 & 1 & 2 & 1.5 & 2 \\
\hline
\end{tabular}

The meteorological data of the real images is listed in table 1 . The calibration factor for each of the five images is related to the fluctuations in the air radiance of $R M S_{a i r}=0.22^{\circ} \mathrm{C}$. As the system response (bit values) is linear with radiance instead of with temperature the final simulator will output radiance values. For simplicity in the present model, we have assumed that the temperature is linear with bit-values. This is only the case for small values (within 5 degrees). In the present model the accuracy is limited. In the reconstruction, whenever an absolute calibration is missing in the images, the air temperature is introduced and the elevation of 0 to 0.1 degrees above the horizon $(1.7 \mathrm{mrad})$ is set to the air temperature.

The average values of the temperature as a function of elevation are obtained from the real image and a smooth fit is stored in a file ('profile\#.dat', 512 points). As noticed before (see section 4) the RMS value of the generated image depends on correlation length. The input RMS values for the generated images therefore are chosen in such a way to match with average RMS values of the real image. At present, the 
input parameters for correlation length, threshold and the multiplying factor are still obtained by visually comparing generated images with the original image. In the analysis we expect to find and adopt an objective approach for the determination of these parameters.

In figure 1 an original and a simulated image are presented. At the left we see the original image that was used to derive the clutter parameters (image 08B5001). At the right the reconstructed image is shown. Note that the images are clearly not identical, but that statistically there is little grey scale difference between them. Note the horizon at about one third from the top of the image.

In figures 2 and 3 the vertical profiles of the temperature of two real and simulated images are presented. There is a general agreement between the profiles of both average temperature and RMS values from the original images and the reconstructed imagery. Detailed differences occur due to the fact that a stochastic process is taken as the input to the regeneration process. Figure 2 corresponds to the images of $08 \mathrm{~B} 5001$ presented in figure 1 . Note the steep slope of about $2{ }^{\circ} \mathrm{C} / \mathrm{mrad}$ in the AVG-figure just below the horizon (the first $1 \mathrm{mrad})$, and the gradual decrease in temperature with increasing elevation in the sky $\left(-0.13{ }^{\circ} \mathrm{C} / \mathrm{mrad}\right.$ at positive elevations $0 \ldots 10 \mathrm{mrad}$ ) and in the sea (negative elevations). The average slope in the sea $(-5 \ldots-20$ mrad) is similar to the one in the sky. The RMS figure shows that the variations in the sky are about twice smaller than the variations in the sea. The sky variations are caused by system noise. Figure 3 shows profiles from the image labeled 264001. The AVG temperature slope in the sky is similar as the one in figure 2. Note that the steep slope just below the horizon (about $1^{\circ} \mathrm{C} / \mathrm{mrad}$ ) is followed by a flat average temperature plane in the sea $(\Delta T / \Delta \epsilon \approx 0$ at $-5 \ldots-20 \mathrm{mrad})$. It is just this fact that makes the modeling effort on a theoretical basis very complicated. Our simulation creates a similar average background as the observed one. The RMS variation in figure 3 shows again more variation in the sea. However, less dominant than in figure 2 (about $50 \%$ more variation in the sea than in the sky).

The modeling effort contains two main areas, an analysis and a simulation. From real imagery recorded under relative or absolute calibration and with local bulk meteo we derive the clutter parameters. This will give us a database of clutter parameters in a bulk meteo environment. The second step is the reconstruction of the imagery based on the clutter data. This latter step requires a re-generation process. The use of the clutter database can be seen as a clata compression process by a factor of 512 .

At the moment the model is in a first evaluation state. By building a large database of clutter and connecting this to the bulk meteo data we will obtain sufficient information to simulate most scenes. We should keep in mind that in general it is very unlikely that the bulk meteo parameters are the only free parameters in the sea background model. During evaluation trials, infrared image data can be recorded with a high performance recording system. These data can then be analyzed and added to the database for the specific scenario. Hence the model will perform well with respect to average background, clutter and contrast values for all data available in the clutter database.

\section{CONCLUSIONS AND FURTHER WORK}

The objective of this study was to provide a model with good functionality in a large number of situations. The present model accurately simulates the statistics of the scenes of which the model is derived. Only a limited number of 10 micron images were analyzed in this short time. In the first period of study, both part of the statistics and the regeneration process needed to be clarified as much as possible. We believe that we have succeeded in doing so, but leaving a large amount of data to be analyzed. We therefore expect the following subjects that need to be studied in the near future in order to develop the final model. 

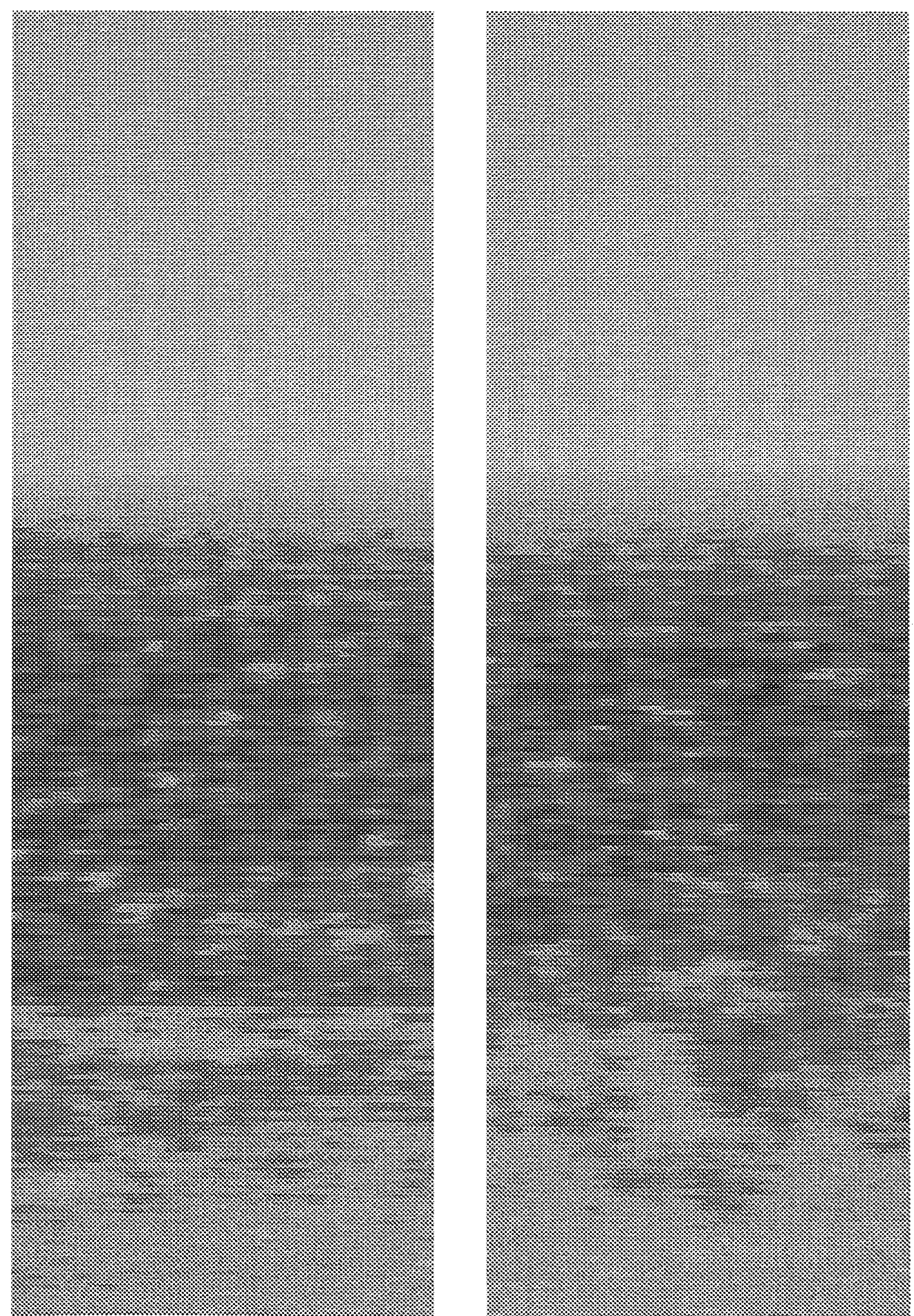

Figure 1: Original image labeled 08B5001 (left) and its simulated image (right). 

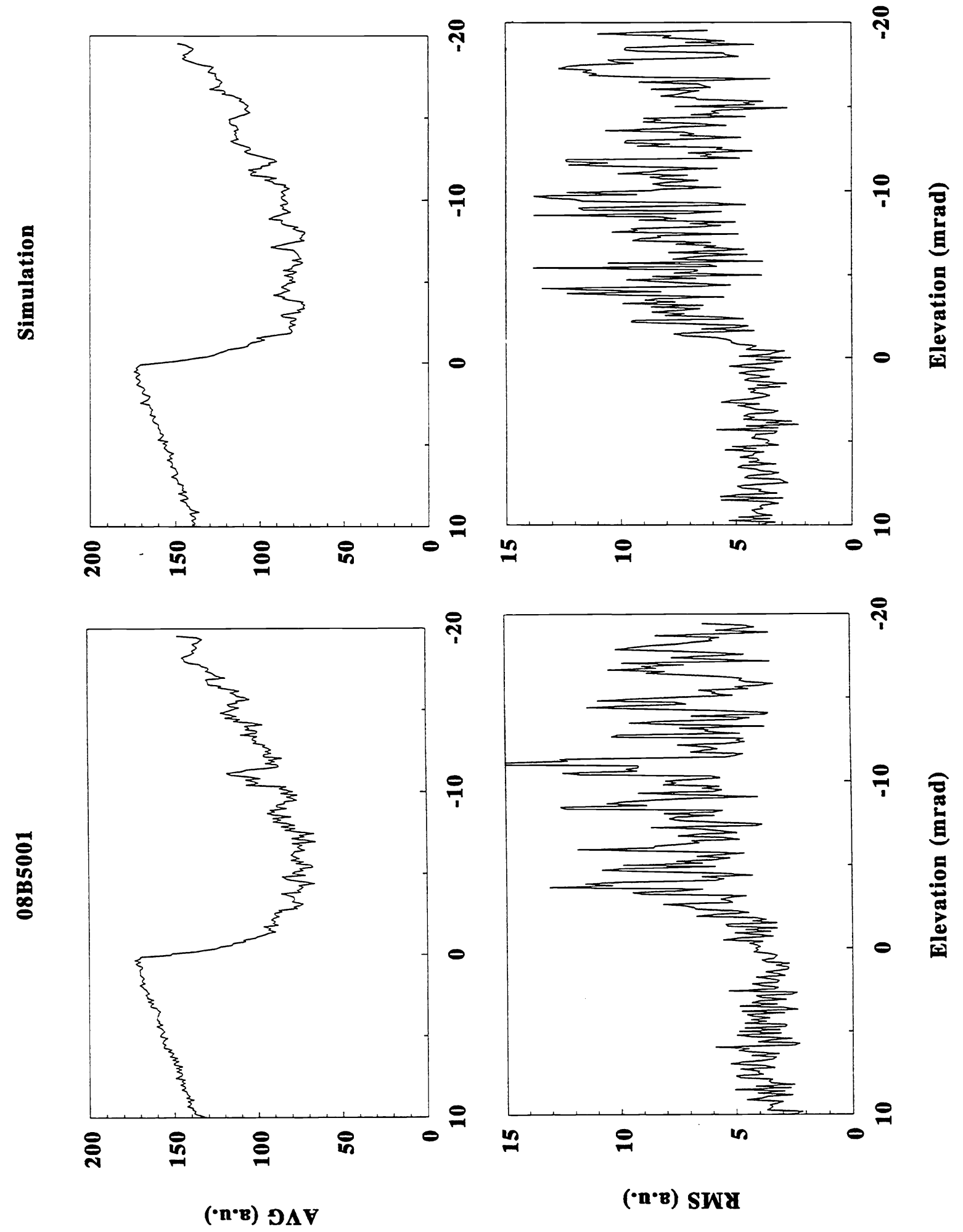

Figure 2: Observed and simulated vertical profiles for the image labeled 08B5001. 

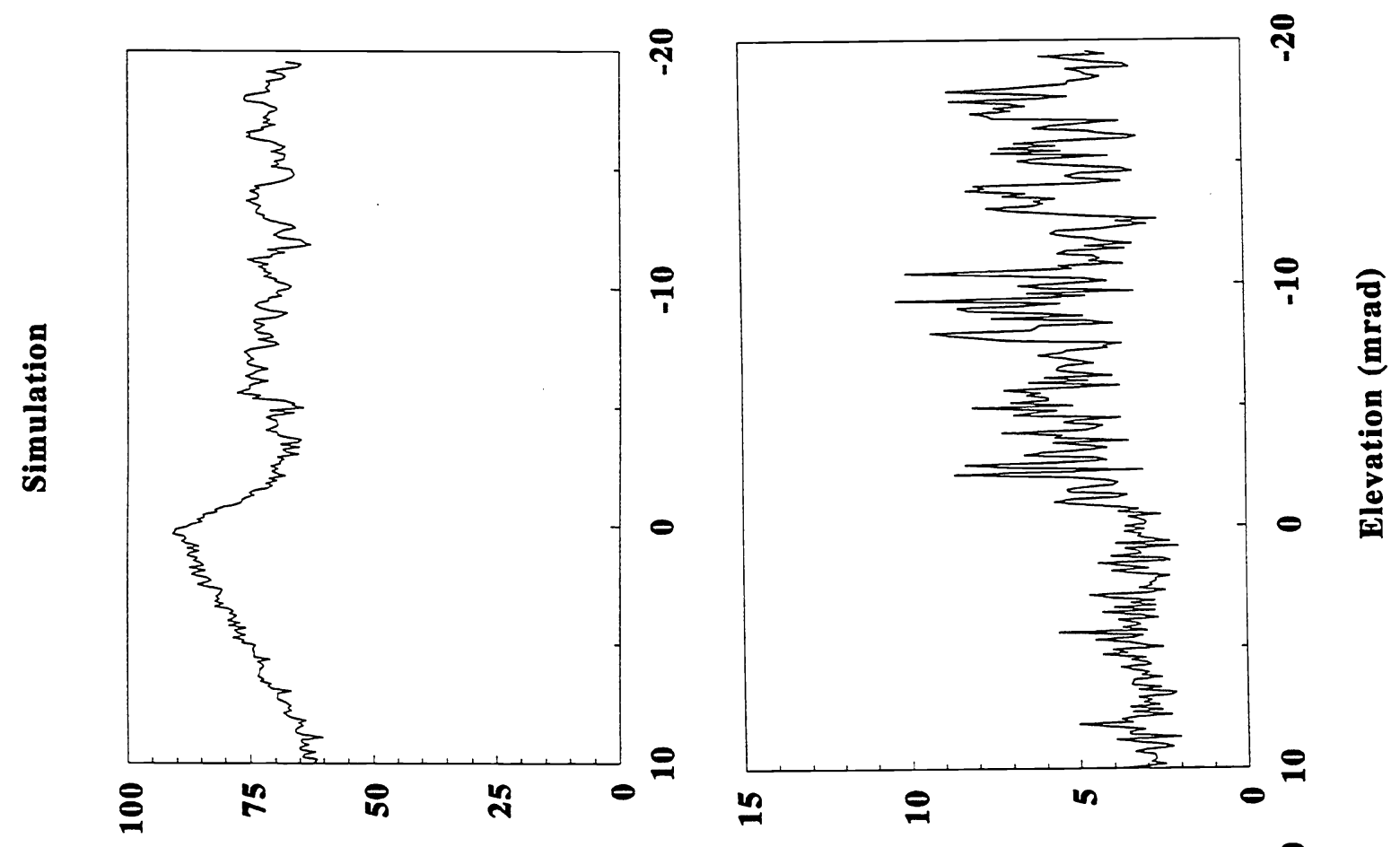

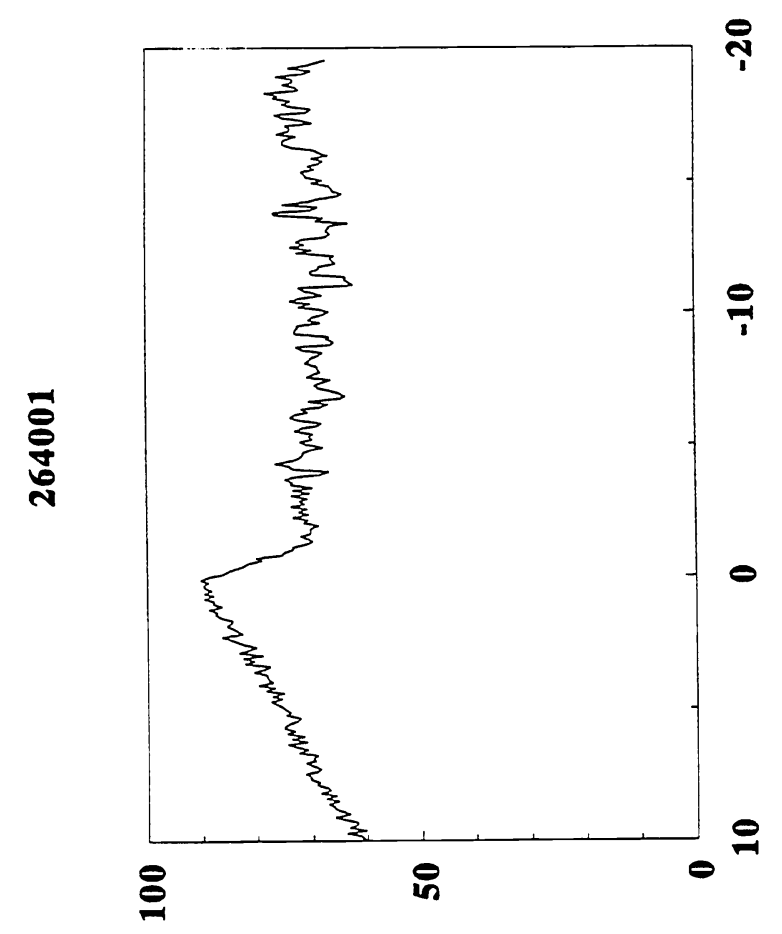

$(\cdot \mathbf{n} \cdot \mathbf{e}) \boldsymbol{D \Lambda} \mathbf{Y}$

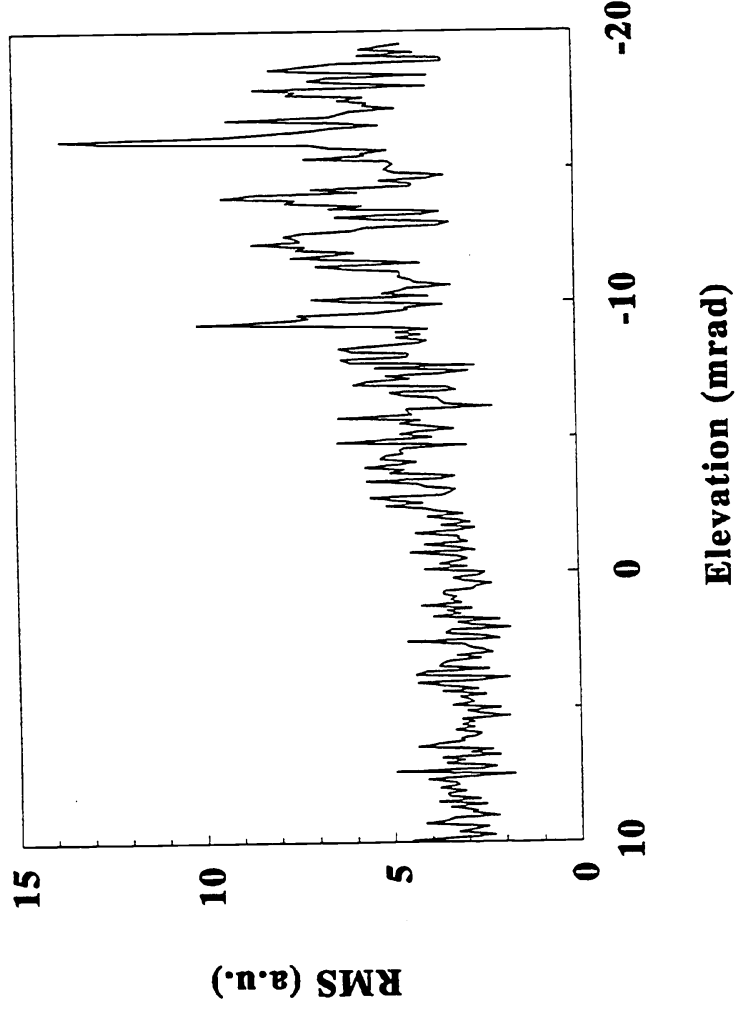

Figure 3: Observed and simulated vertical profiles for the image labeled 264001. 
For normalization purposes a complete change into physical units is to be performed, predicting calibrated radiance values instead of apparent temperatures. In the modeling theory some emphasis has to be placed on checking the re-generation process in more situations. Although it is expected to perform well, other approaches for applying the spatial correlation might be useful (especially in relation to temporal correlation). Some parameters still require a more objective method for calculation.

To provide a validity in more scenarios the clutter database needs to be expanded with more data. Hence more data analysis is needed in both the 5 and the 10 micron bands. Large amounts of data are available for this purpose from trials executed in the last years. This clutter database is actually the physics basis of the model. An integral part of the clutter database is the bulk meteo data. Correlation of the clutter database with the bulk meteo data is an interesting subject. The creation of an interpolator, allowing for clutter predictions in unobserved scenarios would be the extreme expansion of the correlation. Probably not all clutter situations can be derived from the bulk meteo data only.

Additional topics as temporal and spectral effects for the model are under discussion. Temporal correlation effects are under study from image sequences. The spectral resolution of imaging spectrometers might be required in order to do the latter. Finally also special conditions as sun glint will be incorporated in the model.

\section{ACKNOWLEDGMENTS}

I acknowledge the help of Drs. K.W. Benoist in this work. This work is sponsored by the Royal Netherlands Navy under contract A94/KM/787. Analysis work that forms the basis of this paper was sponsored by the US Office of Naval Research (ONR grant N00014-94-1-0405).

\section{REFERENCES}

[1] A. Berk, L.S. Bernstein, D.C. Robertson: 1989, "MODTRAN: A moderate Resolution Model for LOWTRAN7", GL-TR-89-0122, Air Force Geophysics Laboratory, Hanscom AFB, MA (1989)

[2] Schwering P.B.W.: 1992, in "Characterization, Propagation, and Simulation of Sources and Backgrounds $I I^{\prime}$, eds. D. Clement, W.R. Watkins, SPIE Vol. 1687, p:311-322, Orlando Florida (USA), April 20-22, 1992, "Characterization of infrared cloud background clutter"

[3] Schwering P.B.W., Kruseman A.C.: 199:3, in "Characterization, Propagation, and Simulation of Sources and Backgrounds III", eds. D. Clement, W.R. Watkins, SPIE Vol. 1967, paper34, Orlando Florida (USA), April 12-13, 1993: "All-sky infrared cloud clutter analysis"

[4] Schwering P.B.W., Kruseman A.C.: 199:3, in "Infrared Technology XIX", eds. B.F. Andresen, F.D. Shepherd, SPIE Vol. 2020, p108-119, San Diego California (USA), July 12-14, 1993: "All-sky infrared cloud clutter"

[5] M.P. Levesque: 1988, "DREV Cloud Simulation", Ref. 8477-7-PF09/01-SV, Technical Report, SOMAPRO, 1988-10-20

[6] Schwering P.B.W.: 1991, in "Characterization, Propagation, and Simulation of Sources and Backgrounds", eds. D. Clement, W.R. Watkins, SPIE Vol. 1486, p25-36, Orlando Florida (USA), April 2-3, 1991: "Infrared clutter measurements of marine backgrounds"

[7] M.P. Levesque: 1990, "Sea Background. Simulation", DREV R-4569/90, February 1990 
[8] C.R. Zeisse: 1995, "SeaRad, A Sea Radiance Prediction Code", Naval Command, Control and Ocean Surveillance Center, RDT\&E Division, San Diego, CA 92152-5001, Technical Report 1702, November 1995

[9] K.T. Constantikes: 1995, "Modeling and Synthesizing Infrared Ocean Clutter", Johns Hopkins APL Technical Digest, Vol. 16, num. 2 (1995)

[10] P. Saunders: 1968, "Radiance of sea and sky in the infrared window 800-1200 $\mathrm{cm}^{-1}$ ", J. Opt. Soc. Am. $58,645-652(1968)$

[11] C.R. Zeisse: 1995, "Radiance of the ocean horizon", Journal of the Optical Society of America A, p 2022

[12] C.R. Zeisse: 1994, "Radiance of the Ocean Horizon", Naval Command, Control and Ocean Surveillance Center, RDT\&E Division, San Diego, CA 92152-5001, Technical Report 1660, April 1994

[13] IRTool Reference Manual Version 1.4, Arete Associates, February 1995

[14] P.B.W. Schwering, H.A. Lensen: 1996, "Infrared point source behaviour during the MAPTIP trials", TNO-FEL report FEL-94-A.326

[15] P.B.W. Schwering, P.J. Fritz, M. Moerman: 1996, "Infrared point source measurements on board Hr.Ms. Callenburgh", TNO-FEL report, in preparation

[16] T.H. Naylor, J.L. Balintfy, D.S. Burdick, K. Chu: 1986, "Computer Simulation Techniques", John Wiley $\&$ Sons, New York, Chapter 4

[17] N. Ben-Yosef, B. Rahat, G. Feigin: 1983, "Simulation of natural backgrounds", Applied Optics 22, p. 190-193

[18] A. Papoulis: 1965, "Probability, Random Variables, and Stochastic Processes", McGraw-Hill, New York

[19] G.R. Cross, A.K. Jain: 1983, "Markov Random Field Texture Models", IEEE Trans. Pattern Analysis and Machine Intelligence PAMI-5, 1983, p. 25-39 